\title{
Sphingomonas paucimobilis from Blood Stream Infection to Spondylodiscitis
}

\author{
Edmond Puca ${ }^{1 *}$, Arjan Harxhi ${ }^{1}$, Jonida Mehmeti ${ }^{1}$, Arben Rroji ${ }^{2}$, Gentian Huti ${ }^{3}$, Bekim Jata ${ }^{4}$, Albana Daka ${ }^{5}$ and Dhimiter Kraja ${ }^{1}$ \\ ${ }^{1}$ Department of Infection Diseases, University Hospital Center, Tirana, Albania \\ ${ }^{2}$ Department of Radiology, University Hospital Center, Tirana, Albania \\ ${ }^{3}$ Service of Intensive Care Unit, Amerikan Hospital, Tirana, Albania \\ ${ }^{4}$ Department of Cardiology, Amerikan Hospital, Tirana, Albania \\ ${ }^{5}$ Department of Laboratory, Amerikan Hospital, Tirana, Albania \\ "Corresponding author: Edmond Puca, Department of Infection Diseases, University Hospital Center, Tirana, Albania, Tel: 0672058624; E-mail: \\ edmond_puca@yahoo.com
}

Received date: April 20, 2015; Accepted date: May 29, 2015; Published date: June 06, 2015

Copyright: (c) 2015 Puca E, et al. This is an open-access article distributed under the terms of the Creative Commons Attribution License, which permits unrestricted use, distribution, and reproduction in any medium, provided the original author and source are credited.

\begin{abstract}
Sphingomonas paucimobilis, is a yellow-pigmented, aerobic, non-fermentative, gram negative motile bacillus. $S$. paucimobilis which is widely found in nature and hospital environments rarely cause serious or life threatening infections. It's rarely isolated from clinical specimens but it is associated with a great variety of infections in both healthy and immunocompromised people. In this report we present two cases with infection due to S. paucimobilis. One of them was systemic blood stream infection and one focal infection. One was immunocompetent and the other with diabetes mellitus for more than ten years. Both of them were community acquired infection due S. Paucimibilis. These cases are reported to emphasize that $S$. paucimobilis should be kept in mind as a community acquired infection. We present the first cases of Sphingomonas paucimobilis in Albania one of which is a spondylodiscitis in immunocompetent patient and include updated literature concerning infections by this microorganism.
\end{abstract}

Keywords: Sphingomonas paucimobilis, Sepsis; Spondylodiscitis; Infection

\section{Introduction}

Sphyngomonas paucimobilis is a gram-negative, slightly motile with polar flagellum and non-spore-forming, non-fermentative; oxidase positive and forms yellow-pigmented S colony in blood agar, opportunistic pathogen that rarely causes infections in humans $[1,2]$. The microorganism is widely found in natural environment, especially in water and soil [1-11]. Sphingomonas paucimobilis has been associated with variety of infections ranging from milder illness to serious ones. It has been reported to cause outbreaks of bacteremia among immunocompromised patients especially in hematology and oncology [5-8]. On the other hand it can cause infection in nonimmunocompromised patients. Among $S$. paucimobilis nosocomial infections, osteomyelitis is particularly rare: only 2-3 cases of osteomyelitis are recorded in the literature, both in immunosuppressed patients [10-11]. Till now the cases reported of sphingomonas has a very low mortality rate and a good prognosis unlike other gram negative bacteria. We report two cases of community acquired primary bacteremia by Sphingomonas paucimobilis. One of them is a septic shock in an immunocompromised patients and the other is a spondylodiscitis in an immunocompetent. Both of them aren't hospital infections.

\section{Case 1}

A 54-year-old female patient operated 4 years before with aortic valve stenosis, actually with mechanic valve, presented in emergence service with intermittent fever for 3 days, chills, altered mental status, and malaise. She had been suffering from diabetes mellitus for more than 10 years, medicated with oral drugs. During regular visits to the local clinic, the post-prandial finger sugar level always exceeded 200 $\mathrm{mg} / \mathrm{dl}$. On admission the patient was: febrile with fever $39.3^{\circ} \mathrm{C}$, disorientated, with tachycardia, tachypnea, hypotension and oliguria. Upon admission, blood investigations were as follows: white blood cells (WBC) $21.4 \times 10^{6} / \mathrm{mm}^{3}$ (range $4-10 \times 10^{6}$ ), hemoglobin level was $12.3 \mathrm{~g} / \mathrm{dl}$, elevated of hepatic transaminases ALT was $122 \mathrm{UI} / \mathrm{dl}$ (range 0-45) and AST $165 \mathrm{UI} / \mathrm{dl}$ (range 0-35), total bilirubina was $4.74 \mathrm{mg} / \mathrm{dl}$ (normal<1.2), erythrocyte sedimentation rate (ESR) was $52 \mathrm{~mm} / \mathrm{h}$ (range 12-18), fibrinogen $535 \mathrm{mg} / \mathrm{dl}$ (range 200-400), C-reactive protein (CRP) was $23.7 \mathrm{mg} / \mathrm{l}$ (range $<0.5$ ), procalcitonine (PCT) 39.8 $\mathrm{ng} / \mathrm{ml}$ (range $0-2$ ) and $\mathrm{HbA} 1 \mathrm{C} 10.2 \%$. Based on clinical and laboratory data patient was considered as sepsis with unknown pathogen for the moment. In transthorakal and transesophageal echocardiography we excluded vegetations in aortic valve and in others native valve. In total body magnetic resonance imaging (MRI) were seen ischemic lesions in left brain hemisphera, in spleen and in the right kidney. After few hours the patient complicated with acute infarction of inferior myocardial wall and right anterior ventricular wall, fatal arrhythmia, and cardiac arrest. During coronarography we have seen thrombotic lesions in proximal right coronary artery level. We had start treatment with ceftriaxone, metronidasole and moxifloksacine plus supportive therapy. Two sets of hemocultures were taken which both identified $S$.paucimobilis by using Vitek 2 bioMerieux system. Standardized disc diffusion of the organism by the CLSI method showed susceptibility to ceftazidime, ceftriaxone, cefoperazone, cefepime, cefotaxime, ciprofloxacin, moxifloxacin, imipenem, piperacillin-tazobactam, aztreonam, gentamicin, amikacin and trimethoprim/ sulfamethoxazole. Based on antibiogramme result we treated with intravenous imipenem $4 \mathrm{~g}$ /day plus levofloxacin. She responded well to the treatment and discharged on the sixteenth day. 


\section{Case 2}

A 56-year-old previously healthy man with no previous medical illness presented to emergency department at a tertiary hospital in Albania in June 2014 with complaints of severe lumbar pain, significant difficulty in movement, inability in anterior and lateral flexion of vertebral column, numbness of the legs and fever for more than three months. Upon admission, blood investigations were as follows: WBC $7.6 \times 10^{6} / \mathrm{mm}^{3}$, hemoglobin level $10.2 \mathrm{~g} / \mathrm{dl}$ and inflammatory tests as below: CRP $73.1 \mathrm{mg} / \mathrm{dl}$, fibrinogen $627 \mathrm{mg} / \mathrm{dl}$, ESR $55 \mathrm{~mm} / \mathrm{h}$. On CT scan we evidenced: Bone erosion of the intervertebral disc with the generalized and prolabation of lateral sinister disc and foramina in L2-L3. Observed soft tissue edema and iliopsoas muscle at this level without regard for discal hernia. Conclusion L2-L3 spondilodiscitis. On MRI of lumbar colone we observed: Inflammatory lesion with global discal bulbing in L2-L3. Heterogeneous infansement of lesion after iv contrast media administration with bone edema of respective vertebral body's.
Intervertebral disc lesion in L2-L3 vertebral bodies with inflammation in L2 and L3 and paravertebrale soft tissue. Spinal canal and medullar cones in normal position. These images was in favor of the L2-L3 spondylodiscitis. The patient resulted negative for brucellosis, tuberculosis and hemocultures to. In aspiration cultures from vertebral column was isolated: Sphingomonas paucimobilis (with susceptibility to gentamicin, amikacin, ciprofloxacin, moxifloxacin, imipenem, trimethoprim/sulphamethoxazoli, ceftazidime and cefepime). After receiving the antibiogram was initiated therapy with imipenem plus ciprofloxacin for 21 days. After therapy was evidenced clinical improvement of the patient in sense of: significantly reduction of pain, improved mobility, reduction of inflammatory parameters and slightly improved imaging framework. He became outpatient and continued orally therapy for another 4 weeks with ciprofloxacin and trimethoprim/sulphamethoxazole. We followed him for more than 4 months and he was recovered (Figure 1).
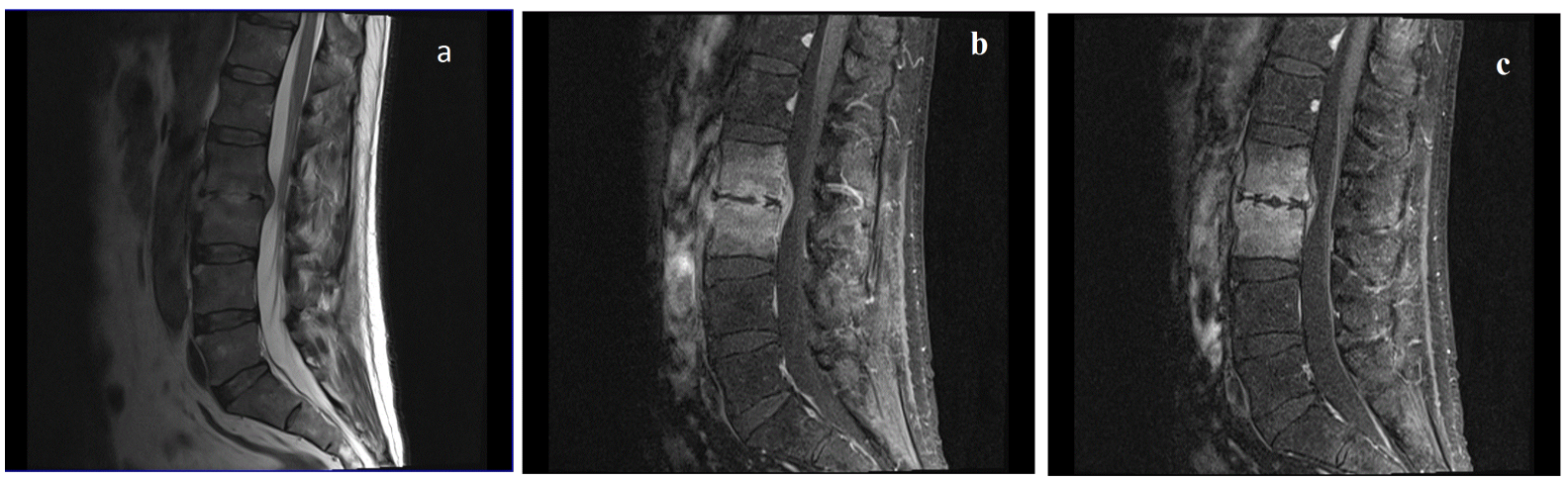

Figure 1: a,b,c. Inflammatory lesion with global discal bulbing in L2-L3. Heterogeneous infansement of lesion after iv contrast media administration with bone edema of respective vertebral body's.

\section{Discussion}

$S$ paucimobilis was first reported to cause human infection in 19775, some others reported first cases in 1979 that masqueraded for years as Pseudomonas paucimobilis [1-7]. It was renamed Sphingomonas paucimobilis in 1990 based on phylogenetic data. One of the best known species of the genus is $S$. paucimobilis, thus named because it has a single polar flagellum with slow motility [12]. The bacteria is widely found in natural environment, especially in water and soil, but it have been isolated from hospital water supplies, respirators, stocked distilled water, blood, wounds, hospital dialysis equipment, patients with meningitis, septicemia, bacteremia, peritonitis, wound infections, soil, river water, deep subsurface sediments, corroding copper pipes, drinking water, rhizosphere and surfaces of plants $[5-7,11]$. Because of its ability to survive in low nutrient conditions, oligotrophic niches, reverse osmosis systems, dialisate and ventilators have been implicated as sources of infection. Another undesirable property of this pathogen is that it is capable of forming a biofilm that can cause metal corrosion of plumbing systems and biofouling of drinking water and industrial water distribution systems [12]. Localized corrosion of copper cold-water pipes results in surface erosions, covered tubercles, and through-wall pinhole pits on the pipe inner surface $[11,13]$. Maybe this ability of it can explain the osteoarticular damage's like in our second case. Human infections caused by $S$. paucimobilis are rare $[5,9,14]$. Since 1977 it has been reported to cause variety of diseases from various parts of the world. This organism is responsible for two types of infection in humans: sporadic or community-acquired infections, probably of endogenous or environmental origin (bacteremia, urinary tract infection, wound infection even meningitis); and nosocomial infections associated with the contamination of sterile fluids employed in hospitals [15]. On the other side it can cause infections in immunocompromised hosts as well as in healthy individuals. Both of our cases presented here, are of community acquired primary bacteremia, as the symptoms appeared before admission to the hospital. One of them (case 1) is a diabetic patient for more than 10 years. She was obese, HbAlC was so high and she treated with oral drugs. Case 2 was an immunocompetent patient, without malignity or chronic diseases. $S$. paucimobilis is an opportunistic pathogen and identification of the organism from clinical specimen is rare. It has been isolated from blood in most of cases presented in literature, sputum, urine, wound, bile, and cerebrospinal fluid. In our cases we identified as $S$. paucimobilis by using Vitek 2 (bioMerieux, France) system. $S$. paucimobilis bacteremia had been reported mainly in patients with indwelling devices or in immunocompromised host, especially those with neutropenia, but our two cases haven't neutropenia $[6,13]$. Infection from $S$. paucimobilis can presented as primary bacteremia, intravascular catheter infections, peritoneal dialysis-associated peritonitis, ventilator-associated pneumonia, biliary tract infection, urinary tract infection, cutaneous 
infection, meningitis, myositis, osteomyelitis, septic arthritis, endophthalmitis, cervical adenitis, bromohidrosis, diarrheal disease and septic shock $[6,7,13,16]$. Primary bacteremia, like in our case 1 , was defined as $S$. paucimobilis bacteremia with signs of sepsis but without an identifiable focus of infection. In our report we have two different cases. One systemic infection and one focal infection. The systemic infection in immunocompromised diabetic patient and the focal infection in immunocompetent patient. And the most important question is; how this pathogen reached to cause a spondylodiscitis? The last is an inflammation of the intervertebral disc and neighboring vertebrae included and vertebral body. Pathogens can infect the spine via three routes: by haematogenous spread, by direct external inoculation, or by spread from contiguous tissues. The haematogenous arterial route is predominant, allowing seeding of infection from distant sites onto the vertebral column. Several blood tests resulted negative in our second case. Multiple studies report predisposing factors like diabetes. Diabetes mellitus is the most commonly identified risk factor, but others include advanced age, malignancy, immunosuppression, injecting drug use, renal failure, rheumatologic disease, liver cirrhosis and previous spinal surgery [6]. In our second cases none of them didn't resulted. The patient didn't do any instrumental examination on vertebral column. He didn't suffer from any diseases before admission on hospital. Belong to the antibiotic therapy, the organism has been reported to be resistant to penicillin and first-generation cephalosporin's because of the production of chromosomally encoded beta-lactamase production and susceptible to tetracycline, chloramphenicol, aminoglycosides, carbapenems, fluroquinolone and trimethoprim/sulphamethaxazole $[6,17,18]$. Aminoglycoside plus a third-generation cephalosporin have been recommended as suitable antibiotics for treatment of $S$. paucimobilis infections in previous study [6]. Carbapenem may be another good treatment choice because of its high susceptibility rate. In our study, it was found to be susceptible to ceftazidime, ceftriaxone, cefoperazone, cefepime, cefotaxime, ciprofloxacin, imipenem, piperacillintazobactam, aztreonam, amikasin and gentamicin and trimethoprim/ sulfamethoxazole. In both of our cases we have used a combination of imipemen plus fluoroquinolones.

\section{Conclusion}

S. paucimobilis is widely distributed in nature and has been isolated from water distribution systems. Although $S$. paucimobilis is a rare hospital infection, it should be kept in mind as a cause, by virtue of the fact that it is a part of the flora in natural environment, especially in patients with immunosuppressive diseases and patients who have other underlying diseases. Thus, even if it is a microorganism of low clinical virulence, its importance cannot be neglected. More importantly this organism has been reported to cause septic shock in immunocompromised patient, and has been increasingly detected recently. The last but no more les important we don't forget it in immunocompetent patient.

\section{References}

1. Dervisoglu E, Meric M, Kalender B, Sengul E (2008) Sphingomonas paucimobilis peritonitis: a case report and literature review. Perit Dial Int 28: 547-550.
2. Tai ML, Velayuthan RD (2014) Sphingomonas paucimobilis: an unusual cause of meningitis-case report. Neurol Med Chir (Tokyo) 54: 337-340.

3. Holmes B, Owen RJ, Evans A, Malnick H, Willcox WR (1977) Pseudomonas paucimobilis, a new species isolated from human clinical specimens, the hospital environment, and other sources. Int J Syst Bacteriol 27: 133-146.

4. Yabuuchi E, Yano I, Oyaizu H, Hashimoto Y, Ezaki T, et al. (1990) Proposals of Sphingomonas paucimobilis gen. nov. and comb. nov., Sphingomonas parapaucimobilis sp. nov., Sphingomonas yanoikuyae sp. nov., Sphingomonas adhaesiva sp. nov., Sphingomonas capsulata comb. nov., and two genospecies of the genus Sphingomonas. Microbiol Immunol 34:99-119.

5. Ã-zdemir M, Pekcan S, Demircili ME, Taşbent FE, Feyzioğlu B, et al. (2011) A rare cause of bacteremia in a pediatric patient with Down syndrome: Sphingomonas paucimobilis. Int J Med Sci 8: 537-539.

6. Toh HS, Tay HT, Kuar WK, Weng TC, Tang HJ, et al. (2011) Risk factors associated with Sphingomonas paucimobilis infection. J Microbiol Immunol Infect 44: 289-295.

7. Ryan MP, Adley CC (2010) Sphingomonas paucimobilis: a persistent Gram-negative nosocomial infectious organism. J Hosp Infect 75: 153-157.

8. Maragakis LL, Chaiwarith R, Srinivasan A, Torriani FJ, Avdic E, et al (2009) Sphingomonas paucimobilis Bloodstream Infections Associated with Contaminated Intravenous Fentanyl1. Emerg Infect Dis 15: 12-18.

9. Charity RM, Foukas AF (2005) Osteomyelitis and secondary septic arthritis caused by Sphingomonas paucimobilis. Infection 33: 93-95.

10. Araújo O, Vidal F, Tapiol J, Richart C (2000) Osteomyelitis caused by Sphingomonas paucimobilis. Enferm Infecc Microbiol Clin 18: 247.

11. White DC, Sutton SD, Ringelberg DB (1996) The genus Sphingomonas: physiology and ecology. Curr Opin Biotechnol 7: 301-306.

12. Gusman V, Medic D, Jelesic Z, Ukropina MM (2012) Sphingomonas paucimobilis as a biofilm producer. Arch Biol Sci Belgrade 64: 1327-1331.

13. Perola O, Nousiainen T, Suomalainen S, Aukee S, Karkkainen UM, et al (2002) Recurrent Sphingomonas paucimobilis-bacteraemia associated with a multi-bacterial water-borne epidemic among neutropenic patients. J Hosp Infect 50: 196e201.

14. Al-Anazi KA, Abu Jafar S, Al-Jasser AM, Al-Shangeeti A, Chaudri NA, et al. (2008) Septic shock caused by Sphingomonas paucimobilis bacteremia in a patient with hematopoietic stem cell transplantation. Transpl Infect Dis 10: 142-144.

15. Reina J, Bassa A, Llompart I, Portela D, Borrell N (1991) Infections with Pseudomonas paucimobilis: report of four cases and review. Rev Infect Dis 13: 1072-1076.

16. Hsueh PR, Teng LJ, Yang PC, Chen YC, Pan HJ, et al. (1998) Nosocomial infections caused by Sphingomonas paucimobilis: clinical features and microbiological characteristics. Clin Infect Dis 26: 676-681.

17. Nandy S, Dudeja M, Das AK, Tiwari R (2013) Community Acquired Bacteremia by Sphingomonas paucimobilis: Two Rare Case Reports. J Clin Diagn Res 7: 2947-2949.

18. Corkill JE, Hart CA, McLennan AG, Aspinall S (1991) Characterization of a beta-lactamase produced by Pseudomonas paucimobilis. J Gen Microbiol 137: 1425-1429. 